\title{
The exact solution of self-consistent equations in the scanning near-field optic microscopy problem
}

\author{
V. Lozovski ${ }^{1}$, S. Bozhevolnyi ${ }^{2}$ \\ ${ }^{1}$ Institute of Semiconductor Physics NAS of Ukraine, \\ 45, prospect Nauki, 252650 Kyiv, Ukraine;Phone/Fax: (+380) 44 2655530; E-mail:lozovski@mol.semicond.kiev.ua \\ ${ }^{2}$ Research Center COM, DTU, Bldg.345 east, DK-2800 Lyngby, Denmark;Fax: (+45)45936581; \\ Phone: (+45)45255763; E-mail: sib@com.dtu.dk
}

\begin{abstract}
The macroscopic approach that allows one to obtain an exact solution of the self-consistent equation of the Lippmann-Schwinger type is developed. The main idea of our method consist in usage of diagram technique for exact summation of the infinite series corresponding to the iteration procedure for solving the self-consistent integral equation. The method developed is applied to calculations of near-field optical images obtained in illumination mode. It is assumed that the system under consideration consists of an object illuminated by the field scattered by a small probe. This assumption allows us to consider multiple scattering between a (point-like) probe and an extended object as well as inside the object. The exact solution for the self-consistent field is then obtained in terms of effective susceptibility of the probe-object system. Application of our method to the description of orientation of molecular complexes at the surface is discussed.
\end{abstract}

Keywords: near-field image, self-consistent equation

Paper received 14.09.99; revised manuscript received 28.09.99; accepted for publication 11.10.99.

\section{Introduction}

Experimental studies of nano-scale systems such as quantum dots, molecular complexes at the surface, etc. have been intensively carried out by numerous research groups in recent years. A very informative tool for high spatial resolution optical measurements is scanning near-field optical microscopy (SNOM). In SNOM, the modifications of electromagnetic radiation, which are caused by the interaction of a SNOM probe with an object smaller than a wavelength of light, are detected. The SNOM technique has been successfully used to image with subwavelenth resolution various surface structures exhibiting different optical contrast mechanisms, such as real and imaginary parts of refractive index, polarization and fluorescence [1]. Quite recently, the first experimental SNOM images related to variations in second-order susceptibilities, i.e. near-field optical images showing nonlinear optical contrast, have been reported $[2,3]$.

Most theoretical papers dealing with the self-consistent problem in near-field optics are based on the consideration of the following linear integral equation (usually called Lippmann-Schwinger equation) [4-6]:

$$
\vec{E}(\vec{R}, \omega)=\vec{E}^{(0)}(\vec{R}, \omega)-i \omega \mu_{0} \int_{V} \overrightarrow{R^{\prime}} \vec{G}\left(\vec{R}, \vec{R}^{\prime}, \omega\right) \vec{\chi}(\omega) \vec{E}\left(\vec{R}^{\prime}, \omega\right)
$$

Here, $\vec{G}\left(\vec{R}, \vec{R}^{\prime}, \omega\right)$ is the Green dyadic of the space in which the particle having the volume $V$ and linear susceptibility tensor $\vec{\chi}(\omega)$ is situated. One of the way for obtaining the solution of Eq.(1) is discretization of Eq.(1) leading to the (self-consistent) system of linear algebraic equations [7]:

$$
\vec{E}\left(\vec{R}_{a}, \omega\right)=\vec{E}^{(0)}\left(\vec{R}_{a}, \omega\right)-i \omega \mu_{0} \sum_{i=1}^{N} \vec{G}\left(\vec{R}_{a}, \vec{R}_{i}, \omega\right) \vec{\chi}(\omega) \vec{E}\left(\vec{R}_{i}, \omega\right)
$$

In Eq.(2) it is implicitly assumed that the self-consistent field is constant inside of each of $N$ subvolumes. The approximation (2) is thereby practically equivalent to the point-like (dipole) approximation. Numerous results were obtained by using Eq.(2) [5, 6, 8-11].

Another way of solving the self-consistent problem (1) consists in using the iteration procedure, i.e., Born approximation of a finite ( $n$-th) order. This procedure implies that multiple scattering inside the system is treated taking into account the scattering processes up to the n-th order. Thus, the first Born approximation $\vec{E}^{(1)}(\vec{R})$ is obtained from Eq.(1) with the incidence field $\vec{E}^{(0)}(\vec{R})$ been substituted into the integrand. This approximation corresponds to the regime of single scattering. To take into account secondary scattered waves one should invoke the second Born approxi- 


\section{Lozovski, S. Bozhevolnyi: The exact solution of self-consistent equations...}

mation, in which the field $\vec{E}^{(1)}(\vec{R})$ found in the first approximation is substituted in the integrand of Eq.(1). In order to include multiple scattered waves of the $n$-th order, one should recurrently carry out the above procedure $n$ times.

It should be emphasized, that method of direct solution of self-consistent problem by numerical solution of the set of the linear algebraic equations (2) is more correct then the method, in which the $n$-th order Born approximation is employed. In order to clarify this point let us rewrite Eq.(1) in the operator form

$$
\vec{E}=\vec{E}^{(0)}+\vec{J} \cdot \vec{E}
$$

with $\vec{J}$ denoting the integral operator in the right part of Eq.(1). The exact solution can then be formally expressed in the form

$$
\vec{E}=(\vec{U}-\vec{J})^{-1} \cdot \vec{E}^{(0)},
$$

where $\overleftrightarrow{U}$ is the unite operator. The $n$-th order Born series expansion becomes

$$
\vec{E}=\vec{E}^{(0)}+\vec{J} \cdot \vec{E}^{(0)}+(\vec{J})^{2} \cdot \vec{E}^{(0)}+\ldots+(\vec{J})^{n} \vec{E}^{(0)} .
$$

This expansion cannot describe the resonant interactions occurring when the (resonant) condition $\operatorname{det}(\vec{U}-\vec{J})=0$ is satisfied. In such a case, any finite order of the Born approximation would not allow one to obtain the exact solution including resonances. To obtain an exact solution of Eq.(1) one has to accomplish exact summation of an infinite series corresponding to the iteration procedure.

The exact solution of the self-consistent integral equation (1) has been reported for some configurations. For the situation, when particle susceptibility is a factorizing function of spatial coordinates, the effective method for direct solution of Eq.(1) was proposed in Ref.[12]. On the other hand, the approach using the diagram technique was proposed for the same model of particle susceptibility in calculations concerned with nano-scale electrodynamics [13].

In the present work we develop the method which is based on the diagram technique for exact solution of a self-consistent equation. This method consists in exact summation of infinite series corresponding to the iteration procedure constructed for the self-consistent Lippmann-Schwin-ger Eq. (1). Note that the field scattered by a probe is included in the iteration procedure.

The proposed technique is demonstrated for general case of spatial dependent susceptibility of a particle. Numerical calculations are provided for simple configuration containing a probe and a sample with spatially independent susceptibilities. Furthermore, in calculations we used the approximation of point-like probe and object in the form of parallelepiped.

\section{Theory}

Let us consider the object and a probe contained in the medium the electro-dynamical properties of which are characterized by the Green function $G_{i j}\left(\vec{R}, \vec{R}^{\prime}, \omega\right)$. Let us suppose that object which, in general, can be a multi-connected body is described by non-local susceptibilities. Namely, the effective current in the object is connected with an electrical field by integral constitutive equation

$$
J_{i}(\vec{R})=\int_{V_{s}} d \vec{R}^{\prime} \chi_{i j}^{(s)}\left(\vec{R}, \vec{R}^{\prime}\right) E_{j}\left(\vec{R}^{\prime}\right)
$$

Integration in Eq.(6) is made over volume of an object which in general case can consist of a few parts (to be multi-connected one). It needs to make the next remark concerning the form of the constitutive Eq. (6). Namely, there exist indications that both linear and non-linear optical susceptibilities can be in general represented in factorizing form, i.e., the kernels of the integral Eq. (6) can be separated on sum of products of functions dependent only on one variable $\vec{R}$ or $\vec{R}^{\prime}$ [14]. In this connection it could mind that in general, the solution of Lippmann-Schwinger equation can be obtained by methods developed for separable susceptibility model (e.g. by the method developed in [12]). But, firstly, the difficulties connected with solution of both «new» constitutive equation and the Lippman-Schwinger equation remain. And, secondly, there is no possibility to obtain a self-consistent solution of Lippmann-Schwinger equation in the case when the susceptibility $\chi_{i j}^{(s)}(\omega)$ is independent on spatial coordinates. Thus, the constitutive equation in the form of Eq.(6) will be used in this work during determination of exact solution of the Lippmann-Schwinger equation. Then, total field in the arbitrary point of the system must obey the equation

$$
E_{i}(\vec{R})=E_{i}^{(0)}(\vec{R})-i \omega \mu_{0} \int_{V_{s}} d \vec{R}^{\prime} \Re_{i j}\left(\vec{R}, \vec{R}^{\prime}\right) \int_{V_{s}} d \vec{R}^{\prime \prime} \chi_{j}^{(s)}\left(\vec{R}^{\prime}, \vec{R}^{\prime \prime}\right) E_{l}\left(\vec{R}^{\prime \prime}\right),
$$

in which, for brevity, the generalized Green function $\mathfrak{R}_{i j}\left(\vec{R}, \vec{R}^{\prime}\right)$ is introduced. This Green function is a photon propagator describing the propagation of photon from point $\vec{R}^{\prime}$ to point $\vec{R}$ of the system.

$$
\begin{aligned}
\Re_{i j}\left(\vec{R}, \vec{R}^{\prime}\right) & =G_{i j}\left(\vec{R}, \vec{R}^{\prime}\right)-i \omega \mu_{0} \int_{V_{P}} d \vec{R}^{\prime \prime} G_{i l}\left(\vec{R}, \vec{R}^{\prime \prime}\right) \times \\
& \times \int_{V_{P}} d \vec{R}^{\prime \prime \prime} \chi_{l k}^{(p)}\left(\vec{R}^{\prime \prime}, \vec{R}^{\prime \prime \prime}\right) G_{l j}\left(\vec{R}^{\prime \prime \prime}, \vec{R}^{\prime}\right)
\end{aligned}
$$

The first term in Eq.(8) describes the direct propagation of photon from point $\vec{R}^{\prime}$ to point $\vec{R}$. The second term of Eq.(8) describes a propagation of photon from point $\vec{R}^{\prime}$ to point $\vec{R}$ of the system by reradiation by the probe with volume $V_{P}$ and susceptibility $\chi_{l k}^{(p)}\left(\vec{R}, \vec{R}^{\prime}\right)$. Eq.(7) could be solved with iteration method. The first step of an iteration procedure is

$$
E_{i}^{(I)}(\vec{R})=E_{i}^{(0)}(\vec{R})
$$

The second step of an iteration procedure is

$$
E_{i}^{(I I)}(\vec{R})=-i \omega \mu_{0} \int_{V_{S}} d \vec{R}^{\prime} \Re_{i j}\left(\vec{R}, \vec{R}^{\prime}\right) \int_{V_{S}} d \vec{R}^{\prime \prime} \chi_{j l}^{(s)}\left(\vec{R}^{\prime}, \vec{R}^{\prime \prime}\right) E_{l}^{(0)}\left(\vec{R}^{\prime \prime}\right)
$$

The third step of an iteration procedure is 


\section{Lozovski, S. Bozhevolnyi: The exact solution of self-consistent equations...}

$$
\begin{aligned}
& E_{i}^{(I I I)}(\vec{R})=-i \omega \mu_{0} \int_{V_{s}} d \vec{R}^{\prime} \Re_{i j}\left(\vec{R}, \vec{R}^{\prime}\right) \int_{V_{s}} d \vec{R}^{\prime \prime} \chi_{j l}^{(s)}\left(\vec{R}^{\prime}, \vec{R}^{\prime \prime}\right)\left(-i \omega \mu_{0}\right) \times \\
& \times \int_{V_{s}} d \vec{R}^{\prime \prime \prime} \Re_{l k}\left(\vec{R}^{\prime \prime}, \vec{R}^{\prime \prime \prime}\right) \int_{V_{s}} d \vec{R}^{I V} \chi_{k m}^{(s)}\left(\vec{R}^{\prime \prime \prime}, \vec{R}^{I V}\right) E_{m}^{(0)}\left(\vec{R}^{I V}\right) .
\end{aligned}
$$

Thus, the exact solution of Eq.(7) can be written as a infinite series of iteration procedure, which in the diagram representation [15] has a form

$E_{i}(\vec{R})=\longrightarrow+\leftrightarrow \longrightarrow+\leftrightarrow \leftrightarrow \longrightarrow+\cdots$,

where the next definitions are used

$E_{i}^{(0)}(\vec{R})=\longrightarrow$,

$-i \omega \mu_{0} \int_{V_{s}} d \vec{R}^{\prime} \Re_{i j}\left(\vec{R}, \vec{R}^{\prime}\right) \int_{V_{s}} d \vec{R}^{\prime \prime} \chi_{j l}^{(s)}\left(\vec{R}^{\prime}, \vec{R}^{\prime \prime}\right) \ldots=\leftrightarrow$

The series (12) could be rewritten in the skeleton diagram representation

$E_{i}(\vec{R})=\longrightarrow+\Leftrightarrow \longrightarrow$,

where operator

$\Leftrightarrow=-i \omega \mu_{0} \int_{V_{s}} d \vec{R}^{\prime} g_{i j}\left(\vec{R}, \vec{R}^{\prime}\right) \int_{V_{s}} d \vec{R}^{\prime \prime} \chi_{j l}^{(s)}\left(\vec{R}^{\prime}, \vec{R}^{\prime \prime}\right)$

containing an unknown function $g_{i j}\left(\vec{R}, \vec{R}^{\prime}\right)$ is represented as an infinite series

$\Leftrightarrow=\leftrightarrow+\leftrightarrow \leftrightarrow+\leftrightarrow \leftrightarrow \leftrightarrow+\ldots$

This series by the next mathematical trick

$\Leftrightarrow=\leftrightarrow+\underbrace{\{\leftrightarrow+\leftrightarrow \leftrightarrow+\ldots\}}_{=\Leftrightarrow} \leftrightarrow$

can be rewritten in the standard Dyson equation form $[15$, 16]

$\Leftrightarrow=\leftrightarrow+\Leftrightarrow \leftrightarrow$.

To find unknown function $g_{i j}\left(\vec{R}, \vec{R}^{\prime}\right)$ let it acts by operator (15) which is obeyed to equation (18) on an arbitrary longrange field. As a result one can obtain the equation

$$
\begin{aligned}
& \int_{V_{S}} d \vec{R}^{\prime} g_{i j}\left(\vec{R}, \vec{R}^{\prime}\right) \int_{V_{s}} d \vec{R}^{\prime \prime} \chi_{j l}^{(s)}\left(\vec{R}^{\prime}, \vec{R}^{\prime \prime}\right) E_{l}^{(A)}\left(\vec{R}^{\prime \prime}\right)=\int_{V_{s}} d \vec{R}^{\prime} \Re_{i j}\left(\vec{R}, \vec{R}^{\prime}\right) \times \\
& \times \int_{V_{s}} d \vec{R}^{\prime \prime} \chi_{j l}^{(s)}\left(\vec{R}^{\prime}, \vec{R}^{\prime \prime}\right) E_{l}^{(A)}\left(\vec{R}^{\prime \prime}\right)+\int_{V_{s}} d \vec{R}^{\prime} g_{i l}\left(\vec{R}, \vec{R}^{\prime}\right) \int_{V_{s}} d \vec{R}^{\prime \prime} \chi_{j l}^{(s)}\left(\vec{R}^{\prime}, \vec{R}^{\prime \prime}\right) \times \\
& \times\left(-i \omega \mu_{0}\right) \int_{V_{s}} d \vec{R}^{\prime \prime} \Re_{l k}\left(\vec{R}^{\prime \prime}, \vec{R}^{\prime \prime}\right) \int_{V_{s}} d \vec{R}^{I V} \chi_{k m}^{(s)}\left(\vec{R}^{\prime \prime}, \vec{R}^{I V}\right) E_{m}^{(A)}\left(\vec{R}^{I V}\right) .
\end{aligned}
$$

Then one can use the fact that long-range arbitrary field slowly changes on the distances of the order of linear dimensions of the system. It means that it is possible to remove the field from the internal integrands in Eq.(19) and use that
$\int_{V} d \vec{R}^{\prime} \vec{\Phi}\left(\vec{R}, \vec{R}^{\prime}\right) \vec{E}^{(A)}\left(\vec{R}^{\prime}\right) \cong \int_{V} d \vec{R}^{\prime} \vec{\Phi}\left(\vec{R}, \vec{R}^{\prime}\right) \cdot \vec{E}^{(A)}(\vec{R})$.

It is followed by

$\int_{V_{S}} d \vec{R}^{\prime}\left\{g_{i j}\left(\vec{R}, \vec{R}^{\prime}\right) \int_{V_{S}} d \vec{R}^{\prime \prime} \chi_{j l}^{(s)}\left(\vec{R}^{\prime}, \vec{R}^{\prime \prime}\right)-g_{i j}\left(\vec{R}, \vec{R}^{\prime}\right)\left(-i \omega \mu_{0}\right) \times\right.$

$\times \int_{V_{s}} d \vec{R}^{\prime \prime} \chi_{j m}^{(s)}\left(\vec{R}^{\prime}, \vec{R}^{\prime \prime}\right) \int_{V_{s}} d \vec{R}^{\prime \prime \prime} \Re_{m n}\left(\vec{R}^{\prime \prime \prime}, \vec{R}^{\prime \prime}\right) \int_{V_{s}} d \vec{R}^{I V} \chi_{n l}^{(s)}\left(\vec{R}^{\prime \prime \prime}, \vec{R}^{I V}\right)-$

$\left.-\Re_{i j}\left(\vec{R}, \vec{R}^{\prime}\right) \int_{V_{s}} d \vec{R}^{\prime \prime} \chi_{j l}^{(s)}\left(\vec{R}^{\prime}, \vec{R}^{\prime \prime}\right)\right\} \cdot E_{l}^{(A)}\left(\vec{R}^{\prime}\right)=0$

Since a field $E_{l}^{(A)}\left(\vec{R}^{\prime}\right)$ in the integrand in Eq.(20) is an arbitrary function of variable $\vec{R}^{\prime}$, to satisfy this equation it needs to be

$$
\begin{aligned}
& g_{i j}\left(\vec{R}, \vec{R}^{\prime}\right) \int_{V_{s}} d \vec{R}^{\prime \prime} \chi_{j l}^{(s)}\left(\vec{R}^{\prime}, \vec{R}^{\prime \prime}\right)-\Re_{i j}\left(\vec{R}, \vec{R}^{\prime}\right) \int_{V_{s}} d \vec{R}^{\prime \prime} \chi_{j l}^{(s)}\left(\vec{R}^{\prime}, \vec{R}^{\prime \prime}\right)- \\
& -g_{i j}\left(\vec{R}, \vec{R}^{\prime}\right)\left(-i \omega \mu_{0}\right) \int_{V_{s}} d \vec{R}^{\prime \prime} \chi_{j m}^{(s)}\left(\vec{R}^{\prime}, \vec{R}^{\prime \prime}\right) \int_{V_{s}} d \vec{R}^{\prime \prime \prime} \Re_{m n}\left(\vec{R}^{\prime \prime \prime}, \vec{R}^{\prime \prime}\right) \times \\
& \times \int_{V_{s}} d \vec{R}^{I V} \chi_{n l}^{(s)}\left(\vec{R}^{\prime \prime \prime}, \vec{R}^{I V}\right)=0
\end{aligned}
$$

Introducing the designations

$M_{j l}\left(\vec{R}^{\prime}\right)=\int_{V_{s}} d \vec{R}^{\prime \prime} \chi_{j l}^{(s)}\left(\vec{R}^{\prime}, \vec{R}^{\prime \prime}\right)$,

and

$$
W_{j l}\left(\vec{R}^{\prime}\right)=\left(-i \omega \mu_{0}\right) \int_{V_{s}} d \vec{R}^{\prime \prime} \chi_{j m}^{(s)}\left(\vec{R}^{\prime}, \vec{R}^{\prime \prime}\right) \int_{V_{s}} d \vec{R}^{\prime \prime} \Re_{m n}\left(\vec{R}^{\prime \prime}, \vec{R}^{\prime \prime}\right) M_{n l}\left(\vec{R}^{\prime \prime}\right),
$$

one can obtain from Eq.(21)

$g_{i l}\left(\vec{R}, \vec{R}^{\prime}\right)=\Re_{i j}\left(\vec{R}, \vec{R}^{\prime}\right) M_{j n}\left(\vec{R}^{\prime}\right)\left[M_{\ln }\left(\vec{R}^{\prime}\right)-W_{\ln }\left(\vec{R}^{\prime}\right)\right]^{-1}$.

Then, from Eqs.(14) and (24) one can find

$$
E_{i}(\vec{R})=\left[\delta_{i j}-i \omega \mu_{0} \int_{V_{s}} d \vec{R}^{\prime} g_{i l}\left(\vec{R}, \vec{R}^{\prime \prime}\right) \int_{V_{s}} d \vec{R}^{\prime \prime} \chi_{l j}^{(s)}\left(\vec{R}^{\prime}, \vec{R}^{\prime \prime}\right)\right] E_{j}^{(0)}(\vec{R}) .
$$

This expression is an exact solution of the LippmannSchwinger equation (7) for the system linear dimensions of which are less then wavelength of external field $E_{i}^{(0)}(\vec{R})$.

\section{The effective susceptibility}

The expression for the self-consistent field in the arbitrary point of a system (Eq.(25)) can be rewritten in terms of ef- 


\section{Lozovski, S. Bozhevolnyi: The exact solution of self-consistent equations...}

fective susceptibility of a system. Namely, using avowed form of the function $g_{i j}\left(\vec{R}, \vec{R}^{\prime}\right)$ it can be obtained from Eq.(25)

$E_{i}(\vec{R})=\left[\delta_{i j}-i \omega \mu_{0} \int_{V_{S}} d \vec{R}^{\prime} \Re_{i l}\left(\vec{R}, \vec{R}^{\prime}\right) \int_{V_{S}} d \vec{R}^{\prime \prime} \mathrm{X}_{l j}\left(\vec{R}^{\prime}, \vec{R}^{\prime \prime}\right)\right] E_{j}^{(0)}(\vec{R})$,

where a tensor

$\mathrm{X}_{l m}\left(\vec{R}^{\prime}, \vec{R}^{\prime \prime}\right)=\left[\delta_{k l}-W_{k n}\left(\vec{R}^{\prime}\right) M_{n l}^{-1}\left(\vec{R}^{\prime}\right)\right]^{-1} \chi_{k m}^{(s)}\left(\vec{R}^{\prime}, \vec{R}^{\prime \prime}\right)$

plays a role of effective susceptibility of the system. As it takes after previous consideration, the value

$$
J_{l}^{(e f f)}\left(\vec{R}^{\prime}\right)=\int_{V_{s}} d \vec{R}^{\prime \prime} \mathrm{X}_{l j}\left(\vec{R}^{\prime}, \vec{R}^{\prime \prime}\right) E_{j}^{(0)}(\vec{R})
$$

is a current inside an object generated by external longrange field. It means that tensor $\mathrm{X}_{l m}\left(\vec{R}^{\prime}, \vec{R}^{\prime \prime}\right)$ is a nonlocal susceptibility, calculated with the account of all scattering processes in the system.

The pole part of this tensor defines the resonances in the system caused by multi-scattering processes into the system. So-called configuration resonances [5] which depends on mutual situation between different parts of a system are described by poles of tensor $\mathrm{X}_{l m}\left(\vec{R}^{\prime}, \vec{R}^{\prime \prime}\right)$, too. These resonances are depended on the dimension and shape of an object. The condition of resonance interaction is determined by usual manner

$$
\operatorname{det}\left[\delta_{k l}-W_{k n}\left(\vec{R}^{\prime}\right) M_{n l}^{-1}\left(\vec{R}^{\prime}\right)\right\rfloor=0 \text {. }
$$

The real and imaginary parts of an effective susceptibility must satisfy the Kramers-Kronig relations [17], which express the causality principle. The real and imaginary parts of $\mathrm{X}_{l m}\left(\vec{R}^{\prime}, \vec{R}^{\prime \prime}\right)$ must obey, moreover, optical theorem conditions [18-20], which express the energy conservation law. Both these fundamental principles can be considered as an examination requirements for effective susceptibility calculated in the framework of determined model.

\section{The separable susceptibility}

There exist a special case of a nonlocal susceptibility factorizing susceptibility. Namely, it can be often supposed that susceptibility of a nano-object has a form $[12,14,21]$

$\chi_{i j}\left(\vec{R}, \vec{R}^{\prime}\right)=-\frac{i a}{\mu_{0} \omega} j_{i}(\vec{R}) j_{j}\left(\vec{R}^{\prime}\right)$

with

$$
a=\mu_{0} \sum_{m, n} \frac{f_{m}-f_{n}}{\hbar(\omega+i v)+E_{m}-E_{n}} .
$$

Where $j_{i}(\vec{R})$ and $j_{i}\left(\vec{R}^{\prime}\right)$ are the transition current densities between one-electron eigenstates $m$ and $n$ calculated at the space points $\vec{R}$ and $\vec{R}^{\prime}$, respectively. The quantities $f_{m}$ and $f_{n}$ are Fermi-Dirac distribution factors giving the probabilities that the states $m$ and $n$ are occupied in thermal equilibrium $[12,21]$.

For simplicity used was the two-level model susceptibility of an object containing only transitions between $n=$ 0 and $m=1$ states. Then, using Eqs (22) and (23) one obtains

$M_{j l}\left(\vec{R}^{\prime}\right)=-\frac{i a}{\mu_{0} \omega} j_{j}\left(\vec{R}^{\prime}\right) \gamma_{l}^{(0)}$,

and

$W_{j l}\left(\vec{R}^{\prime}\right)=-\frac{i a}{\omega \mu_{0}} j_{j}\left(\vec{R}^{\prime}\right) a N \gamma_{l}^{(0)}$,

where

$\gamma_{l}^{(0)}=\int_{V_{s}} d \vec{R} j_{l}(\vec{R})$.

In according to Ref.[12], let us introduce a parameter

$N=\int_{V_{s}} d \vec{R}^{\prime \prime} j_{m}\left(\vec{R}^{\prime \prime}\right) \int_{V_{s}} d \vec{R}^{\prime \prime \prime} \Re_{m n}\left(\vec{R}^{\prime \prime \prime}, \vec{R}^{\prime \prime}\right) j_{n}\left(\vec{R}^{\prime \prime \prime}\right)$,

then, substituting Eqs.(32)-(35) into Eq.(24), one obtains

$g_{i l}\left(\vec{R}, \vec{R}^{\prime}\right)=\Re_{i j}\left(\vec{R}, \vec{R}^{\prime}\right) j_{j}\left(\vec{R}^{\prime}\right) \gamma_{n}^{(0)}\left(j_{l}\left(\vec{R}^{\prime}\right) \gamma_{n}^{(0)}\right)^{-1}[1-a N]^{-1}$.

Using the next equality

$j_{j}\left(\vec{R}^{\prime}\right) \gamma_{n}^{(0)}\left(j_{l}\left(\vec{R}^{\prime}\right) \gamma_{n}^{(0)}\right)^{-1}=\delta_{j l}$,

from Eq.(36) we can derive an expression

$g_{i l}\left(\vec{R}, \vec{R}^{\prime}\right)=\Re_{i j}\left(\vec{R}, \vec{R}^{\prime}\right)[1-a N]^{-1}$.

At last, substitution of Eq.(38) into expression for selfconsistent field (27) gives the expression for a local field of the system characterized by nonlocal susceptibility (30). Namely,

$E_{i}(\vec{R})=\left[\delta_{i j}(\vec{R})-a \frac{1}{1-a N} F_{i}(\vec{R}) \gamma_{j}^{(0)}\right] E_{j}^{(0)}\left(\vec{R}_{p}\right)$,

where expression in the brackets plays the role of so-called local field factor [12], and

$F_{i}(\vec{R})=\int_{V_{S}} d \vec{R}^{\prime} \Re_{i l}\left(\vec{R}, \vec{R}^{\prime}\right) j_{l}\left(\vec{R}^{\prime}\right)$.

As it is easily seen, Eqs (39) and (40) are the expressions obtained earlier (See, e.g., Ref.[12]) with other method.

\section{The system consisting of a small probe and an object characterized by susceptibility independent on spatial coordinates}

Let us consider the system consisting of an object with linear dimensions less then wavelength of an external field and small probe which could be considered as a point-like particle. Let us assume that probe is small as compared with characteristic wavelength of a field. It means that for arbitrary long-range field the equation for reradiated field by a probe can be written in the form (Cf. Eq.(8))

SQO, 2(3), 1999 


\section{Lozovski, S. Bozhevolnyi: The exact solution of self-consistent equations...}

$$
\begin{aligned}
E_{i}^{(R)}(\vec{R}) & =-i \omega \mu_{0} \int_{V_{p}} d \vec{R}^{\prime} G_{i l}\left(\vec{R}, \vec{R}^{\prime}\right) \int_{V_{p}} d \vec{R}^{\prime \prime} \chi_{l j}^{(p)}\left(\vec{R}^{\prime}, \vec{R}^{\prime \prime}\right) E_{j}^{(A)}\left(\vec{R}^{\prime \prime}\right) \cong \\
& \cong G_{i j}\left(\vec{R}, \vec{R}_{p}\right) E_{j}^{(A)}\left(\vec{R}_{p}\right)
\end{aligned}
$$

where

$$
\overline{G_{i j}\left(\vec{R}, \vec{R}_{p}\right)}=-i \omega \mu_{0} \int_{V_{p}} d \vec{R}^{\prime} G_{i l}\left(\vec{R}, \vec{R}^{\prime}\right) \int_{V_{p}} d \vec{R}^{\prime \prime} \chi_{l j}^{(p)}\left(\vec{R}^{\prime}, \vec{R}^{\prime \prime}\right) .
$$

It means that the generalized Green function describing the electrodynamical properties of the media in which an object situated have to consist of two parts

$$
\Re_{i j}\left(\vec{R}, \vec{R}^{\prime}\right)=G_{i j}\left(\vec{R}, \vec{R}^{\prime}\right)+\overline{G_{i l}\left(\vec{R}, \vec{R}_{p}\right)} \cdot G_{l j}\left(\vec{R}_{p}, \vec{R}^{\prime}\right),
$$

This Green function is a photon propagator describing the propagation of photon from point $\vec{R}^{\prime}$ to point $\vec{R}$ of the system. The first term of Eq.(43) describes the direct propagation of a photon from point $\vec{R}^{\prime}$ to point $\vec{R}$. The second term describes propagation of a photon from point $\vec{R}^{\prime}$ to point $\vec{R}$, which is re-radiated by a point-like probe situated at point $\vec{R}_{p}$. In the case of independent on spatial coordinate susceptibility of an object and a small probe, Eq.(26) can be rewritten as

$E_{i}(\vec{R})=\left[I_{i j}(\vec{R})-i \omega \mu_{0} \int_{V_{s}} d \vec{R}^{\prime} \Re_{i l}\left(\vec{R}, \vec{R}^{\prime}\right) \mathrm{X}_{l m}\left(\vec{R}^{\prime}\right) I_{m j}\left(\vec{R}^{\prime}\right)\right] E_{j}^{(0)}\left(\vec{R}_{p}\right)$,

with effective susceptibility of a system

$$
\mathrm{X}_{i j}(\vec{R})=\left[\left(\chi_{i j}^{(s)}\right)^{-1}-D_{j i}(\vec{R})\right]^{-1} \text {, }
$$

and «incoming field» tensor

$$
I_{i j}(\vec{R})=\delta_{i j}+\overline{G_{i j}\left(\vec{R}, \vec{R}_{p}\right)} .
$$

In Eq.(45) the designation

$$
D_{i j}(\vec{R})=-i \omega \mu_{0} \int_{V_{s}} d \vec{R}^{\prime} \Re_{i j}\left(\vec{R}, \vec{R}^{\prime}\right)
$$

is used for the self-energy part. It should be emphasized that because generalized Green function is in the integrand of Eq.(47), self-energy part describes all scattering processes including scattering of the field between a probe and an object.

These equations will be used for numerical calculations of near-field image of an object in the next part of the work.

\section{Numerical calculations}

To illustrate the developed method we calculated the image of near-field for the system consisting of rectangular dielectric parallelepiped and small spherical probe. For simplicity, it is supposed that susceptibilities of an object and a probe are independent on spatial coordinates. It means that a probe is rather small for the approximation (40) to be valid. Calculation was made for a model shown in Fig.1. It is supposed that a small probe is scanned along the scanning plane. A probe re-radiates the external field acting on the system. Due to action of both external and re-radiated fields on the object the currents are generated inside the object. These currents cause radiation, which is detected as a near-field image. The dependence of intensity of near-field image on a probe position in the scanning plane was calculated. Cartesian coordinate system was chosen with origin situated at the center of an object.

In recent years the interest to nano-technologies based not only on solid state materials but on molecular technologies is permanently increased $[22,23]$. In this connection the problem of molecular or molecular complexes orientation at the surface becomes important. There is a big number of the papers devoted both to theoretical and experimental studies of the problem of orientation of molecules and molecular clusters at the surface $[24,25]$. On the other hand, the SNOM technique allows to obtain different images of different oriented nanostructures [26]. These facts allows us to propose this new method of calculation of nearfield image to problem of termining orientation of nanoobjects with respect to axes of external field polarization. These reasons were taken into account when three types of objects are used in the calculations: an object of the 1-st type is characterized by dimensions $40 \times 200 \times 20 \mathrm{~nm}^{3}$, with a short side along $\mathrm{O} z$ axis. An object of the 2-nd type is characterized by dimensions $400 \times 200 \times 20 \mathrm{~nm}^{3}$, with a short side along $\mathrm{O} z$ axis. And object of the 3 -d type is characterized by dimensions $200 \times 200 \times 20 \mathrm{~nm}^{3}$, with a short side along $\mathrm{O} z$ axis. Radius of a probe is much less then wavelength $r_{0}<<\lambda$, namely $r_{0}=4 \mathrm{~nm}, \lambda=800 \mathrm{~nm}$. Due to these circumstances the near-field and middle-filed parts of the

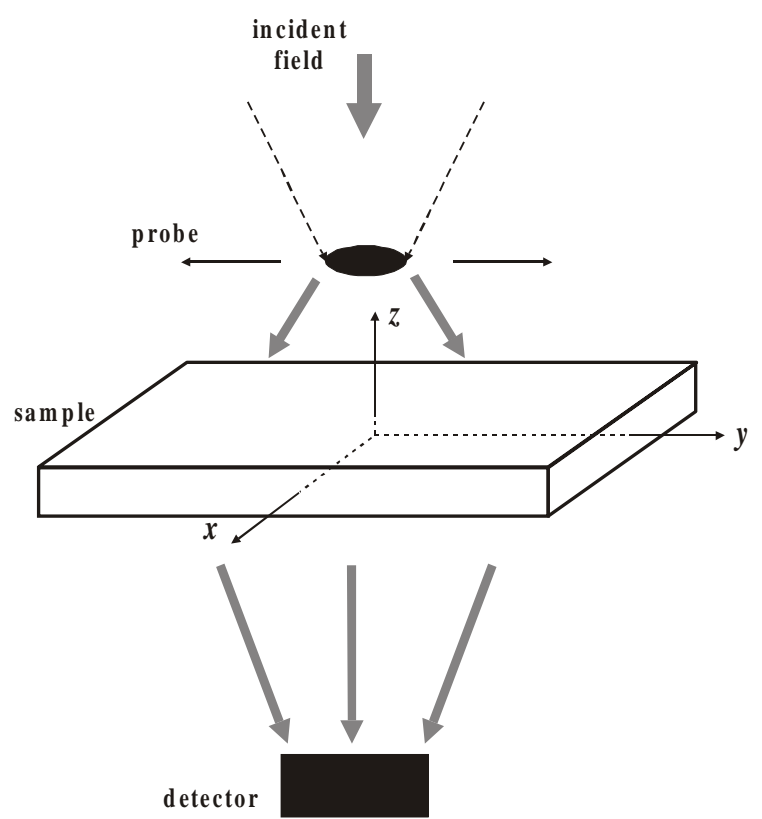

Fig.1. The scheme of SNOM experiment for which the calculations were made in the work. 


\section{Lozovski, S. Bozhevolnyi: The exact solution of self-consistent equations...}

photon propagator were used in numerical calculations [5, 12]. Namely,

$$
\begin{aligned}
& \vec{G}\left(\vec{R}, \vec{R}^{\prime}\right)=\frac{1}{4 \pi}\left[\left(-\frac{i c}{\omega R_{d}^{3}}+\frac{c^{2}}{\omega^{2} R_{d}^{3}}\right) \cdot \vec{U}+\right. \\
& \left.+\left(\frac{3 i c}{\omega R_{d}^{2}}-\frac{3 c^{2}}{\omega^{2} R_{d}^{2}}\right) \cdot \vec{e}_{R_{d}} \vec{e}_{R_{d}}\right] e^{i(\omega / c) R_{d}}
\end{aligned}
$$

with $\vec{U}$ unit dyadic, $\vec{R}_{d}=\vec{R}-\vec{R}^{\prime}, R=\left|\vec{R}-\vec{R}^{\prime}\right|$ and $\vec{e}_{R}=\vec{R} / R$.

Since the external field supposed as monochromatic one, dielectric constants of both probe and object were supposed to be constant and equal to $\varepsilon_{p r}=2.25$ and $\varepsilon=3$ respectively.

Calculated was the value, which characterizes the intensity of a light forming the near-field image in far zone. It is clear that this intensity is equal to

$$
J=\left|-\frac{\chi_{E}}{4 \pi} \int_{V_{s}} \vec{G}_{F-F}\left(\vec{R}, \vec{R}^{\prime}\right) \vec{E}\left(\vec{R}^{\prime}\right)\right|^{2}
$$

with $\vec{G}_{F-F}\left(\vec{R}, \vec{R}^{\prime}\right)$ far-field part of the Green dyadic. Since the distance at which the near-field image is detected, $D_{r}$, is much more than a linear dimension of an object, one can write

$$
J=\left|-\frac{\chi_{E}}{4 \pi} \vec{G}_{F-F}\left(\vec{R}, \vec{R}_{0}\right)\right|^{2}\left|\int_{V_{s}} d \vec{R}^{\prime} \vec{E}\left(\vec{R}^{\prime}\right)\right|^{2}
$$

with $R_{0}$ coordinate of the center of an object. So, due to the distance $D_{r}=\left|\vec{R}-\vec{R}_{0}\right|$ that is constant in the defined experiment, the intensity of light which forms the near-field image can be written as

$$
J=\text { Const } \cdot\left|\int_{V_{s}} d \overrightarrow{R^{\prime}} \vec{E}\left(\vec{R}^{\prime}\right)\right|^{2},
$$

This value is calculated in the present work. In Eq. (51) $\vec{E}(\vec{R})$ is the self-consistent field calculated in according to Eq.(44).

In the numerical calculations the problem of divergence of integrals at $\vec{R}-\vec{R}^{\prime}=0$ arises. For solution this problem it is necessary to use the approach developed in the works of van Bladel [26] and Yaghjian [27] in which the idea of exclusive volume was discussed. This approach consist in the formal procedure

$$
\vec{E}(\vec{R})=-i \omega \mu_{0} \lim _{\delta \rightarrow 0} \int_{V_{s}-V_{\delta}} \vec{G}\left(\vec{R}, \vec{R}^{\prime}\right) \cdot \vec{J}\left(\vec{R}^{\prime}\right) d \vec{R}^{\prime}-\frac{\vec{L} \cdot \vec{J}(\vec{R})}{i \omega \mu_{0}},
$$

where $\vec{L}$ is a source dyadic which depends solely on the geometry of a «principal volume», $V_{\delta}$, which excludes the singularity of $\vec{G}$ and becomes infinitesimally small in the limit as its maximum chord length $\delta$ approaches to zero [27]. The dyadic $\vec{L}$ for a rectangular parallelepiped was calculated in [28] and is equal to $\frac{1}{4 \pi}\left(\Omega_{x} \vec{e}_{x} \vec{e}_{x}+\Omega_{y} \vec{e}_{y} \vec{e}_{y}+\Omega_{z} \vec{e}_{z} \vec{e}_{z}\right)$ with

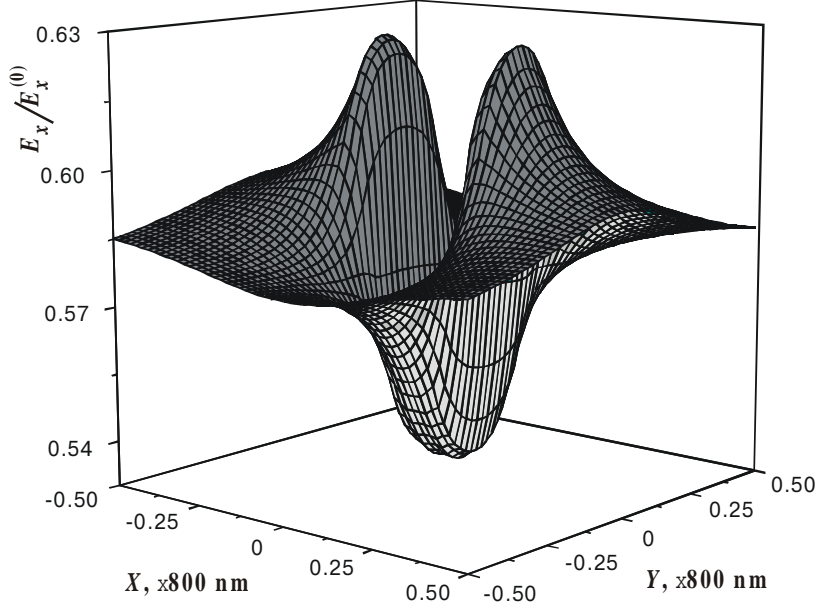

a

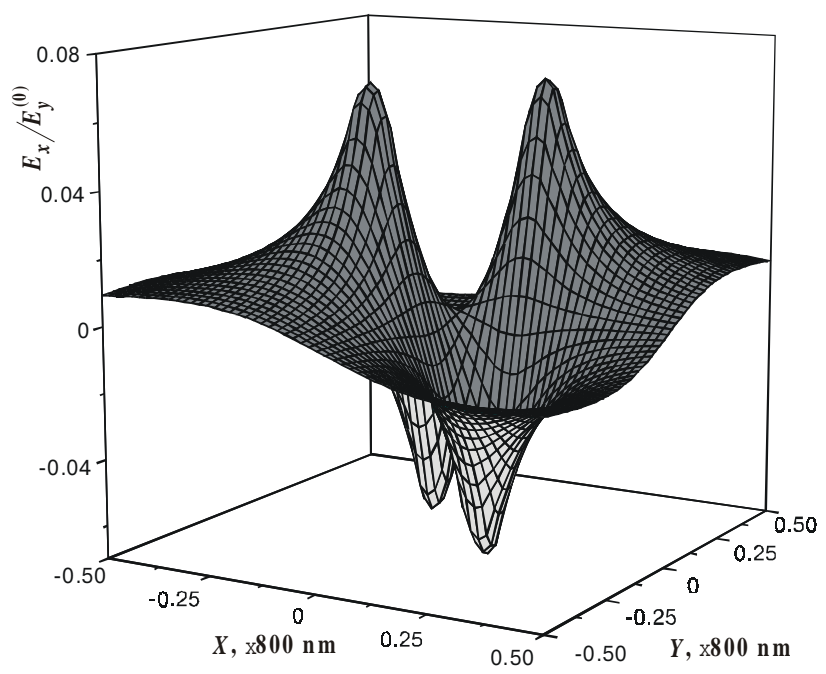

b

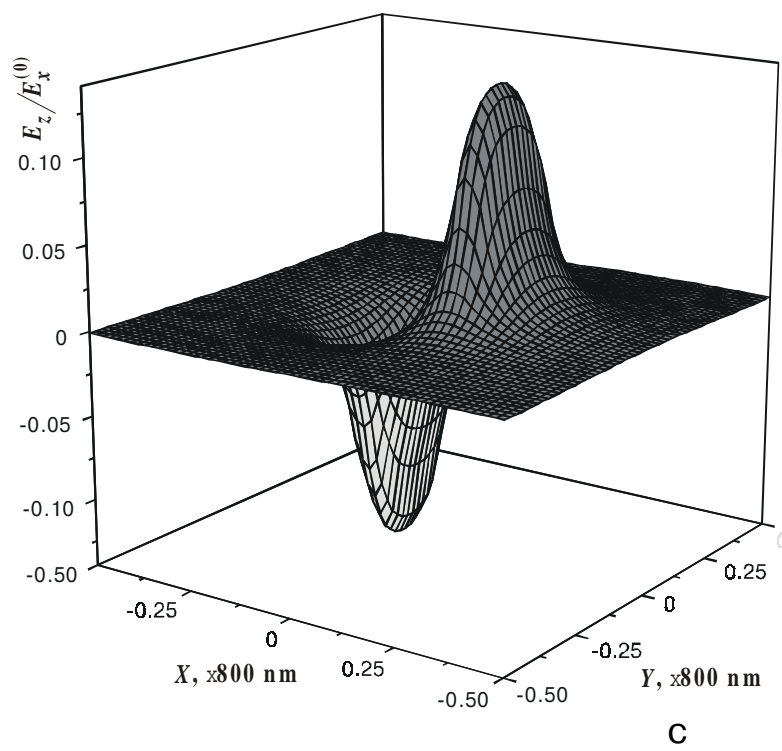

Fig.2. The 3d-field distribution at the probe scanning along scanning plane situated at the distance of $30 \mathrm{~nm}$ from an object that is rectangular bulk with dimensions $\left(200 \times 200 \times 20 \mathrm{~nm}^{3}\right)$. 


\section{Lozovski, S. Bozhevolnyi: The exact solution of self-consistent equations...}

$\Omega_{x}, \Omega_{y}, \Omega_{z}$ twice the solid angle subtended by a side perpendicular to the $x, y, z$ direction, respectively $\left(\Omega_{x}+\Omega_{y}+\Omega_{z}=4 \pi\right)$ [27].

The near-field images were calculated for different values of distance $l$ between a scanning plane and an object. The main attention is paid to the influence of mutual orientations of an object and external field polarization on formation of near-field object image.

The first series of calculations was made for stick-like object (the 1-type object). This model calculation can give
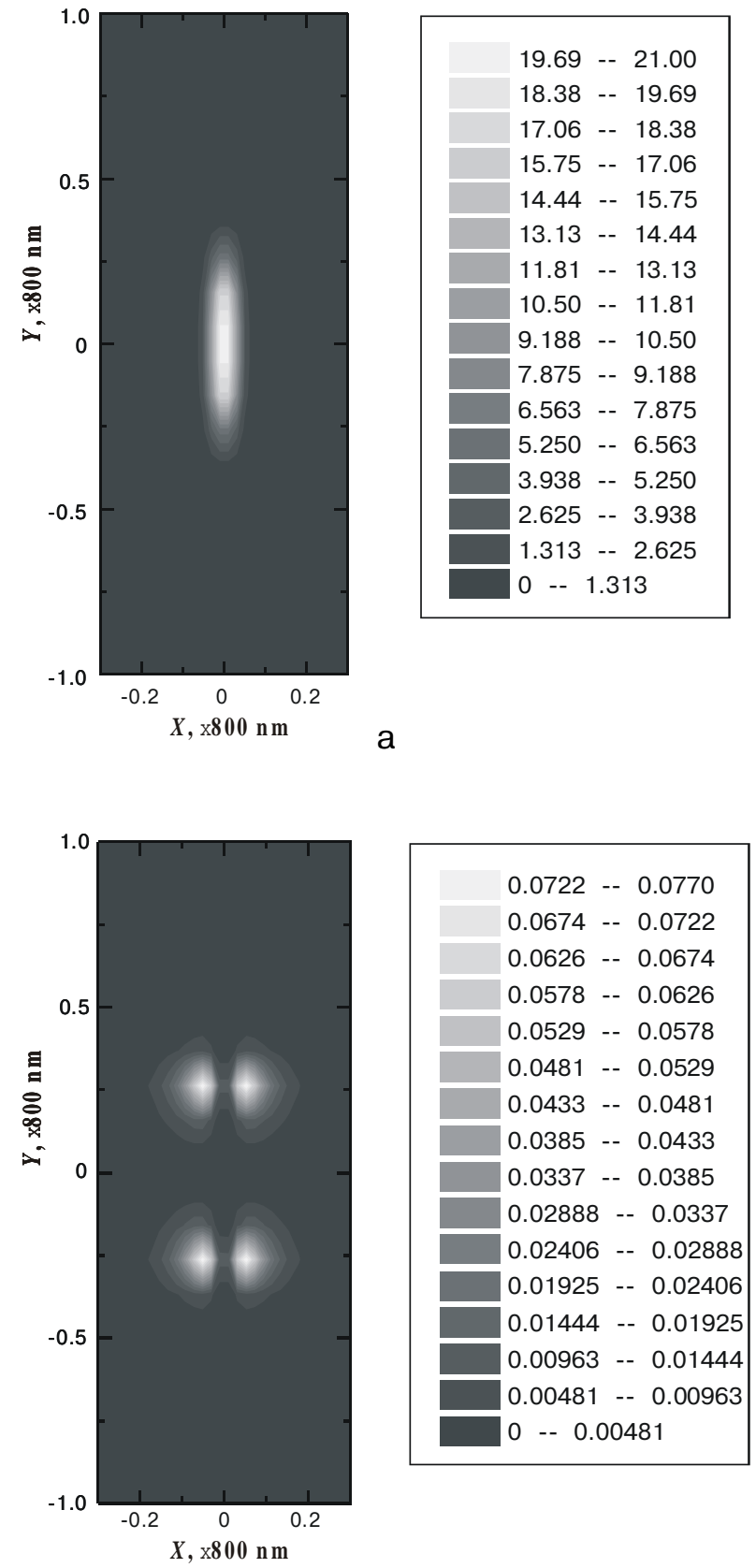

C the notion about a possible near-field images of stick-like polymers or molecular complexes. The results of these calculations are shown in Figs 2-5. It needs to be pointed that increasing of the distance between scanning plane and an object leads not only to some smearing out the image and decreasing of intensity of image. In the case of external light polarization along a long axes of an object the near-field image can have rather complicated structure (see, Fig.5) and very similar to near-field image given by linear chain of a dipoles.
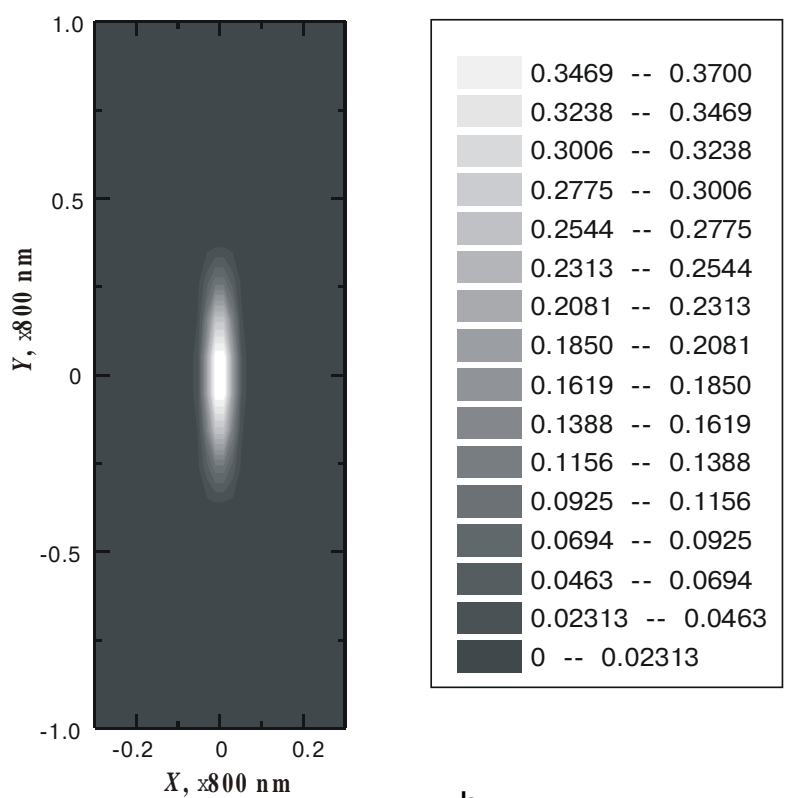

b
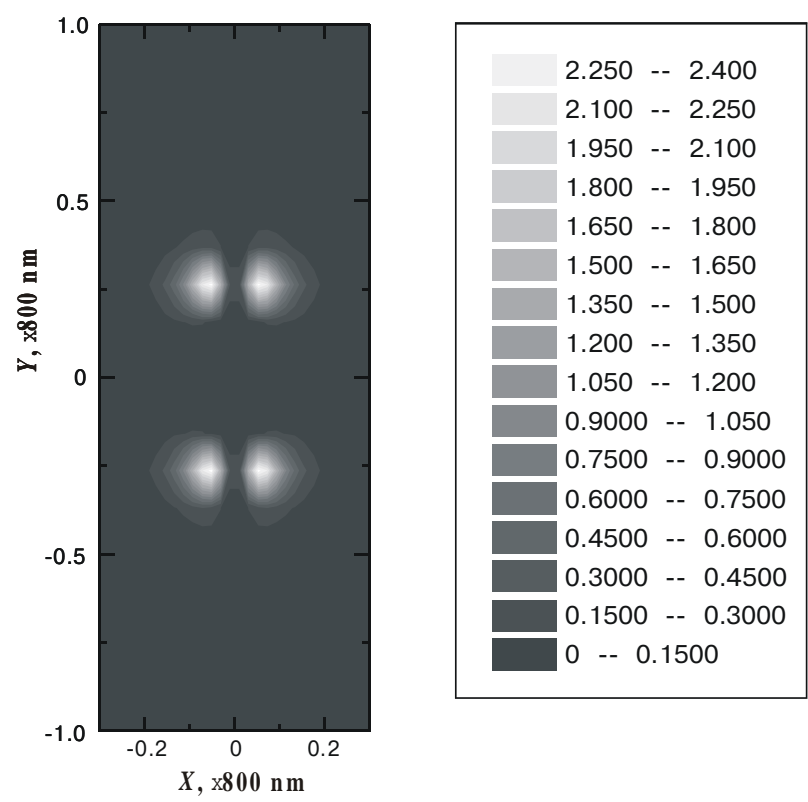

d

Fig.3. The gray scale map representation of near-field image calculations for stick-like object $\left(80 \times 400 \times 20 \mathrm{~nm}^{3}\right)$ when the distance between a scanned plane and an object is equal to $30 \mathrm{~nm}$. (a) - both external and detected fields are polarized along Ox axes; (b) - both external and detected fields are polarized along $\mathrm{O} y$ axes; (c) - the external field is polarized along $\mathrm{O} x$ axes and detected field is polarized along Oy axes; (d) - the external field is polarized along $\mathrm{O} y$ axes and detected field is polarized along $\mathrm{O} x$ axes. 


\section{Lozovski, S. Bozhevolnyi: The exact solution of self-consistent equations...}

The results of calculations of a near-field image of rectangular bulk 2-nd type object are shown in Figs 6 and 7. The results of the same calculations for rectangular bulk 3-d type object are shown in Figs.8 and 9. All these results allows to hope that polarimetric measurements of near-field images of an objects having rectangular form of an projection on the scanning plane, can give a possibility for determining orientation of an object with respective to external field polarization direction. It needs to be emphasized that near-field images of cross-excited fields (e.g., external field is polarized along $\mathrm{O} x$ axes and detected field is polarized along $\mathrm{O} y$ axes and vice versa) have a specific structure consisting of four bright smears at the corners of an object projection on the scanning plane. This characteristic behavior of near-field images could be observed in all calculated examples.
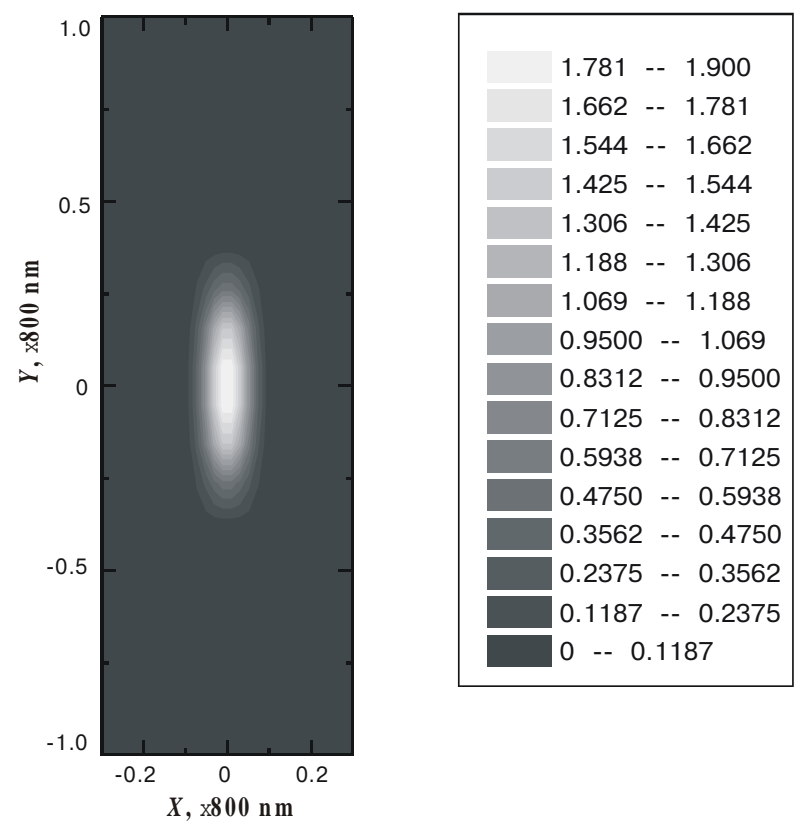

a

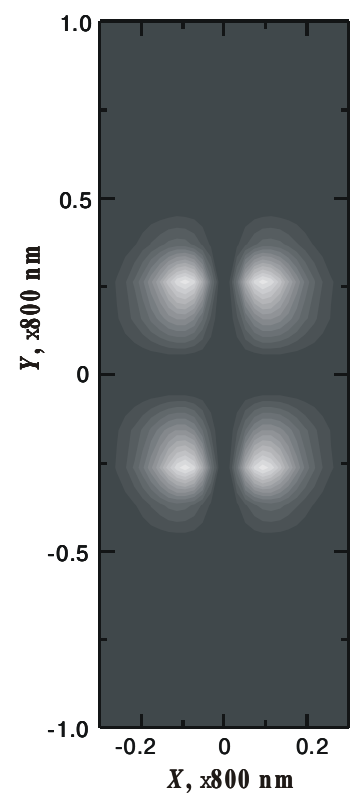

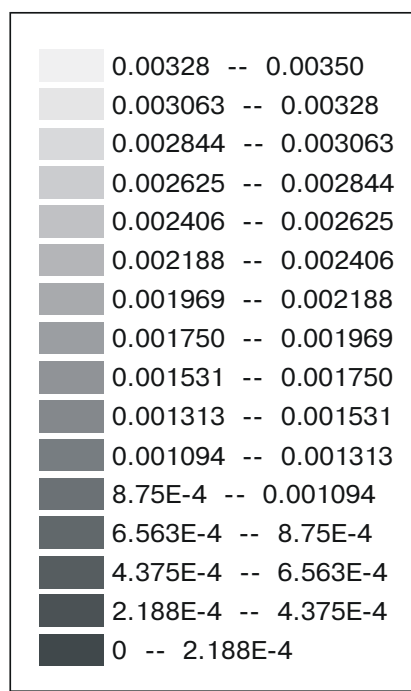
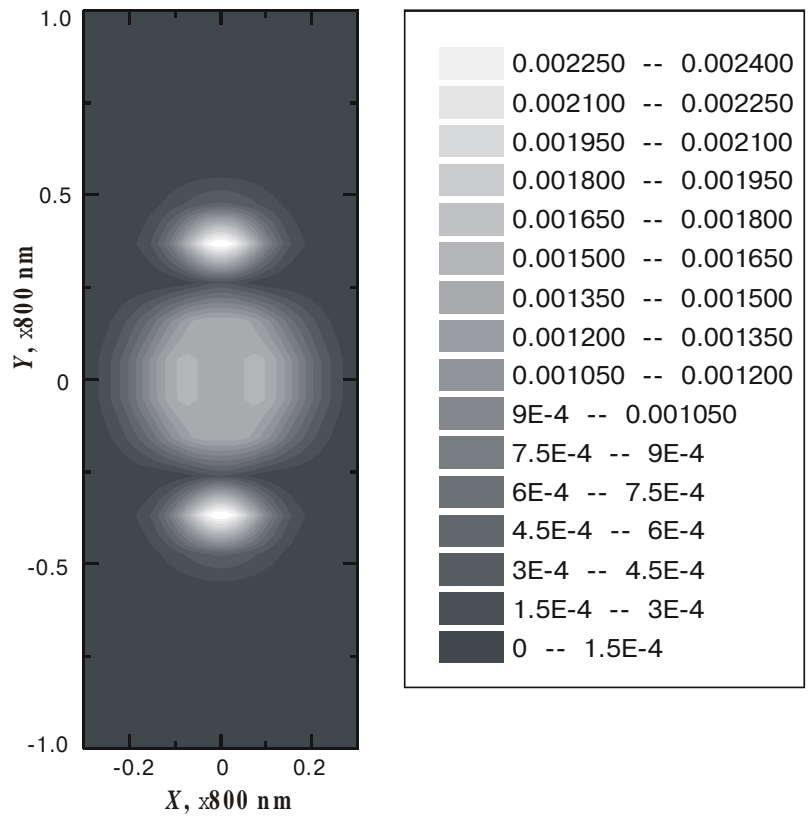

b

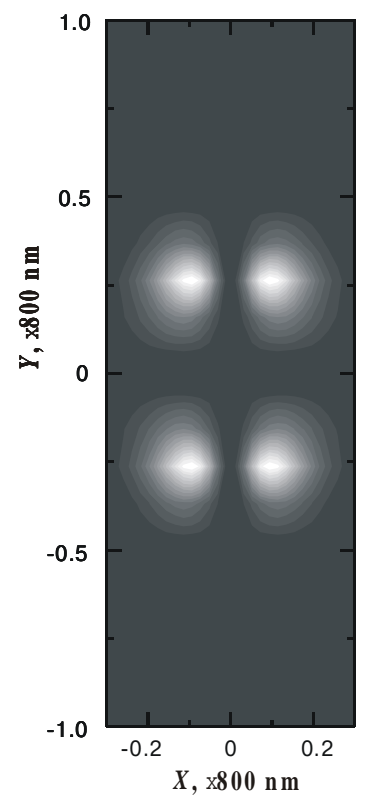

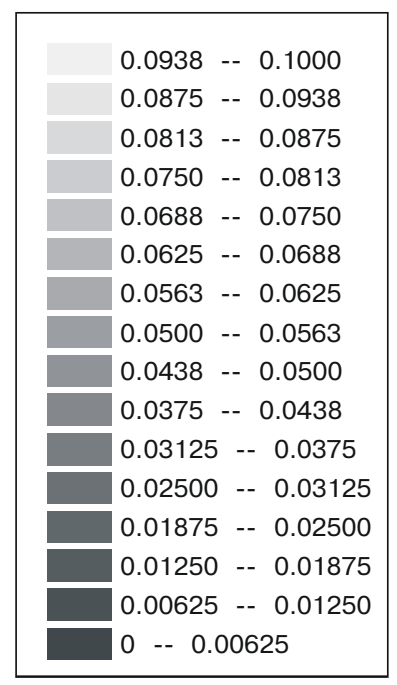

d

C

Fig.4. The same as in Fig.2, but calculated for distance between a scanned plane and an object $l=90 \mathrm{~nm}$. 


\section{Lozovski, S. Bozhevolnyi: The exact solution of self-consistent equations...}

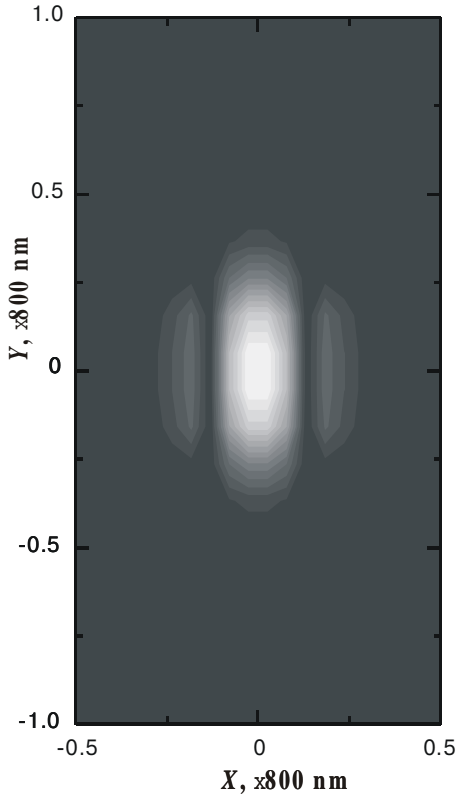

a

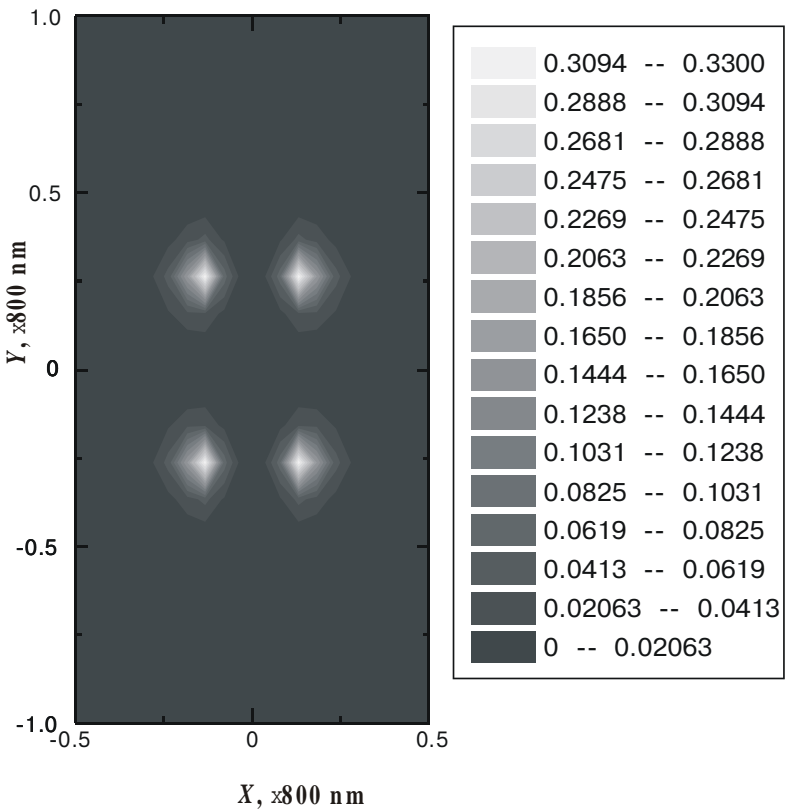

C

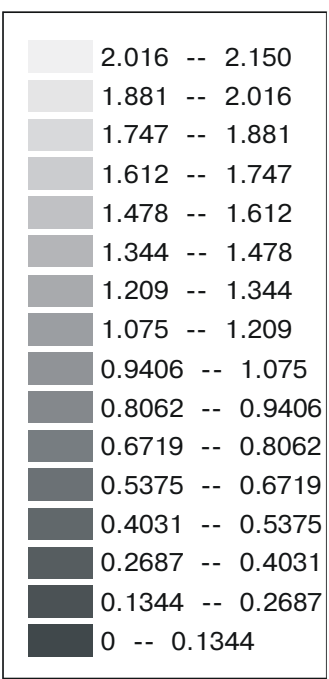

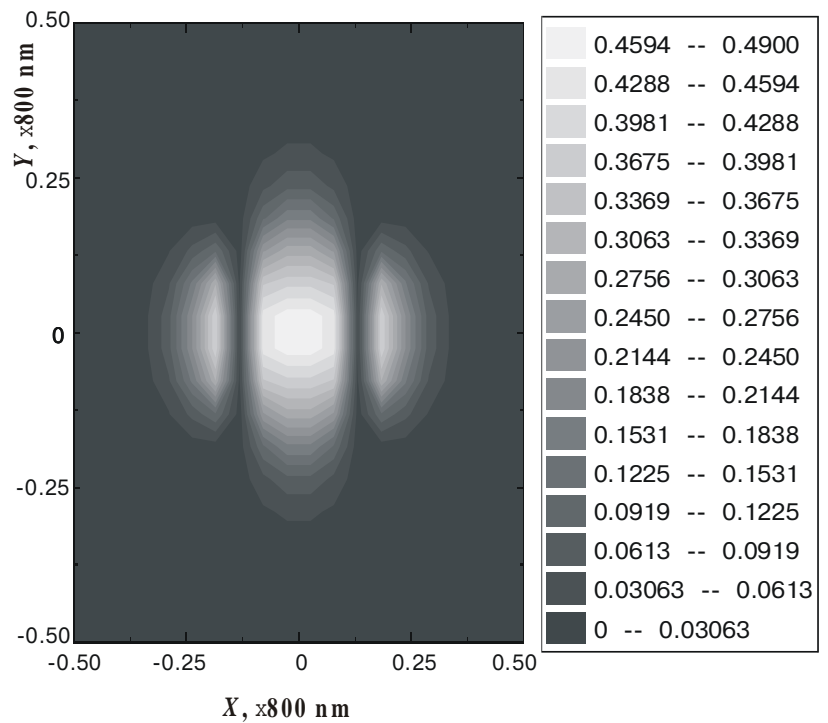

b
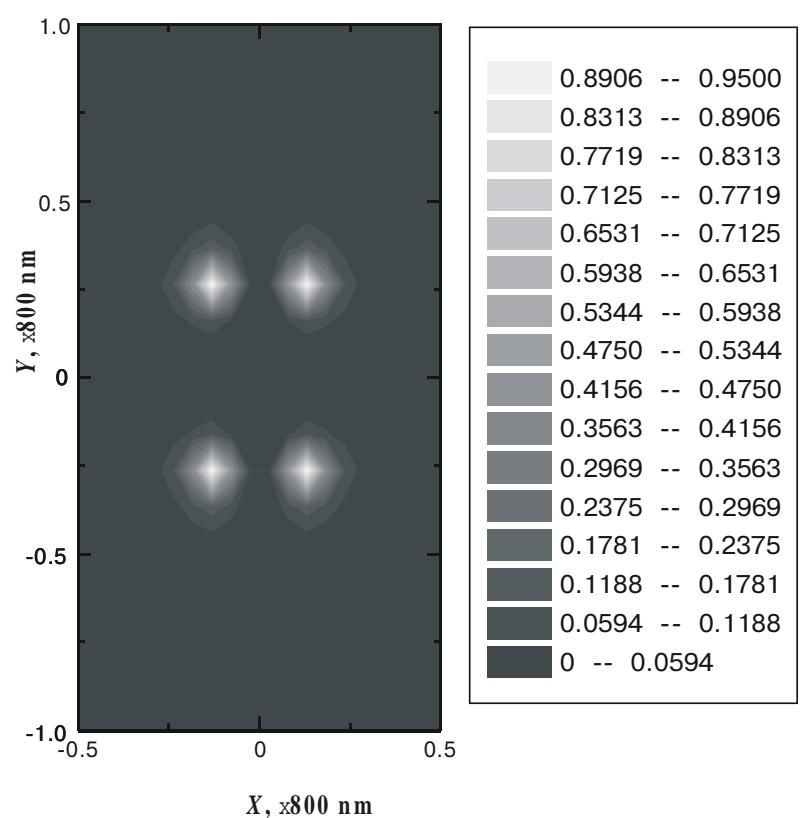

d

Fig.5. The gray scale map representation of near-field image calculations an rectangular object $\left(200 \times 400 \times 20 \mathrm{~nm}^{3}\right)$ when the distance between a scanned plane and an object is equal to $30 \mathrm{~nm}$. (a) - both external and detected fields are polarized along $\mathrm{O} x$ axes; (b) - both external and detected fields are polarized along Oy axes; (c) - the external field is polarized along $\mathrm{O} x$ axes and detected field is polarized along $\mathrm{O} y$ axes; (d) - the external field is polarized along $\mathrm{O} y$ axes and detected field is polarized along $\mathrm{O} x$ axes. 
V. Lozovski, S. Bozhevolnyi: The exact solution of self-consistent equations...

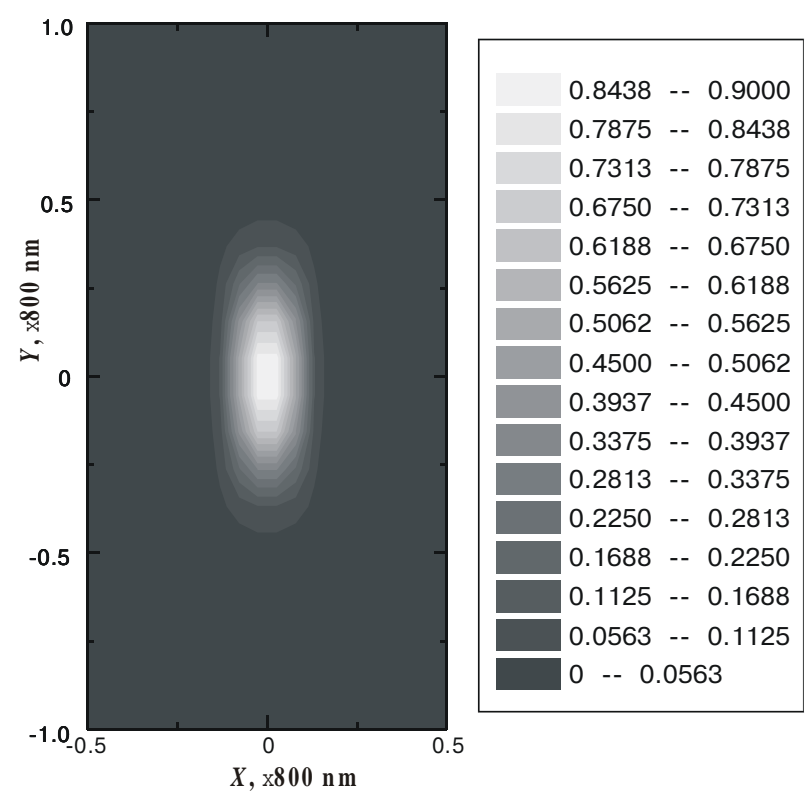

a

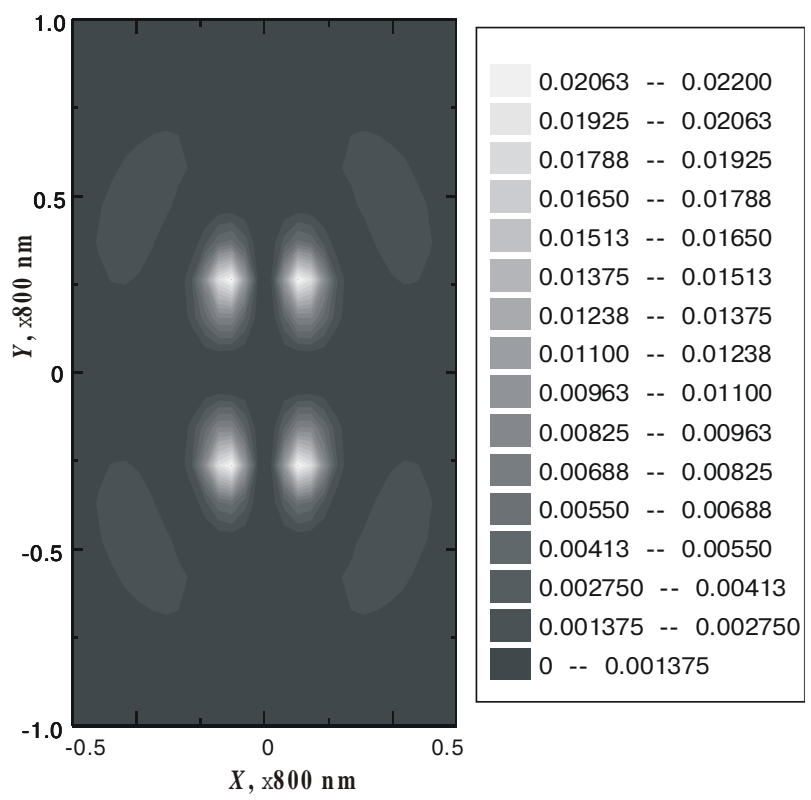

C
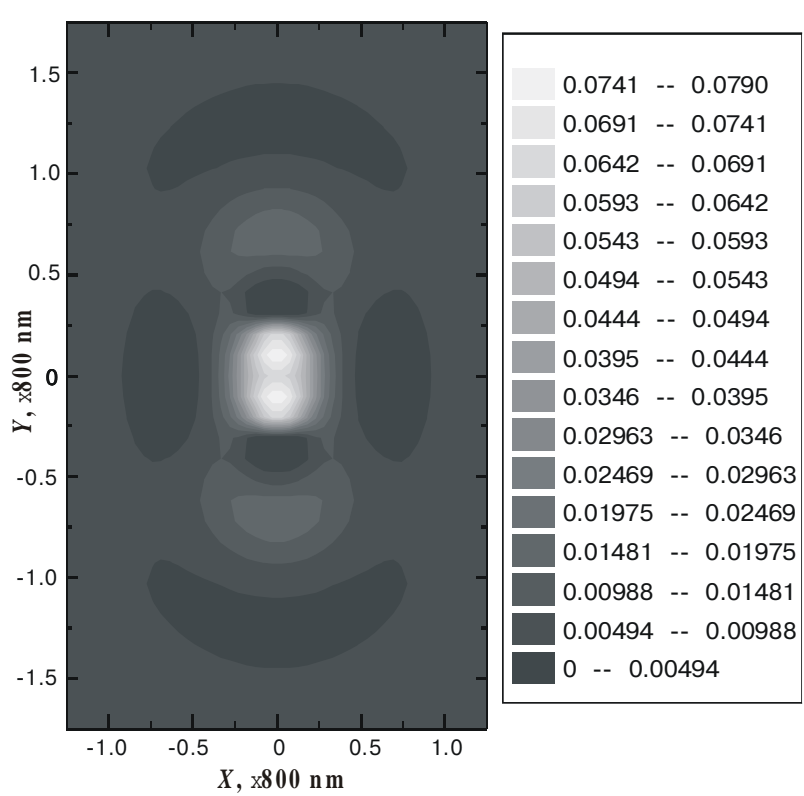

b

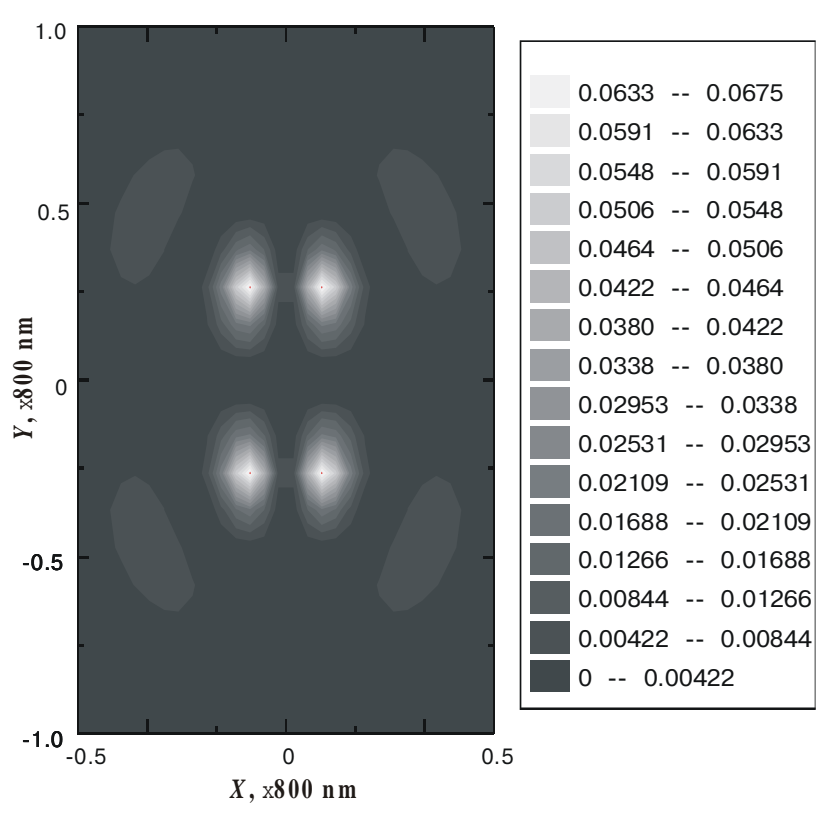

d

Fig.6. The same as in Fig.4, but calculated for distance between a scanned plane and an object $l=90 \mathrm{~nm}$. 


\section{Lozovski, S. Bozhevolnyi: The exact solution of self-consistent equations...}
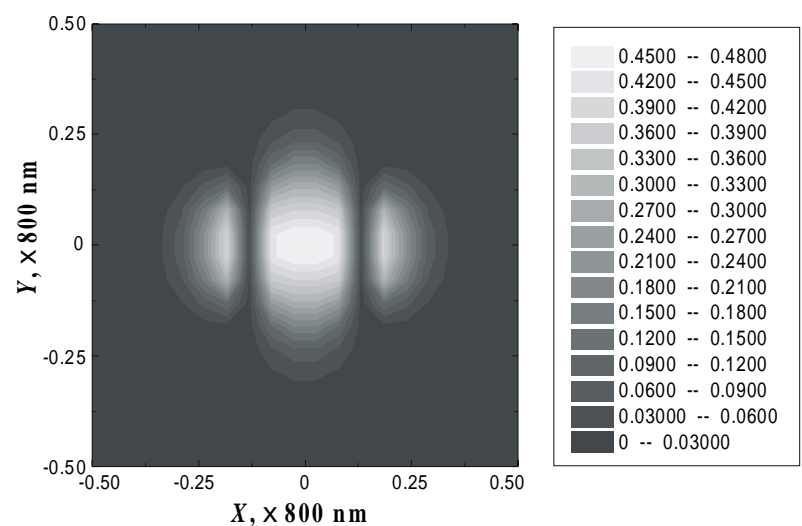

a

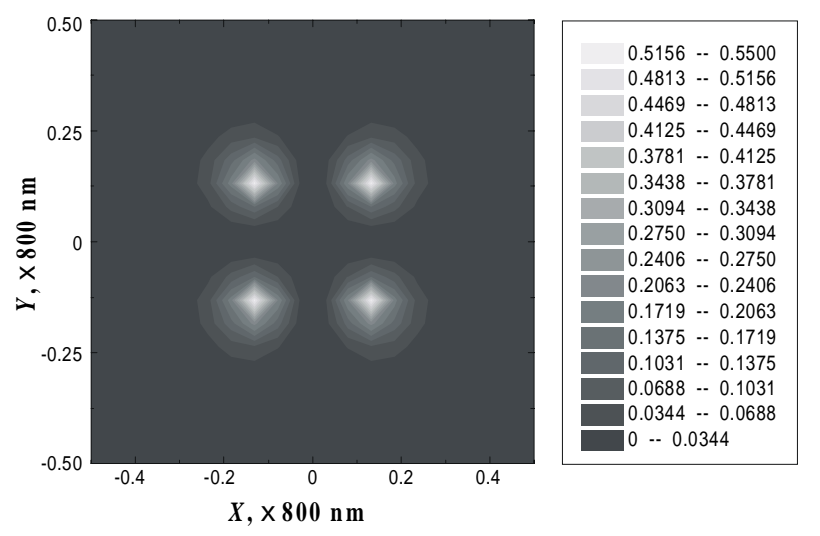

C
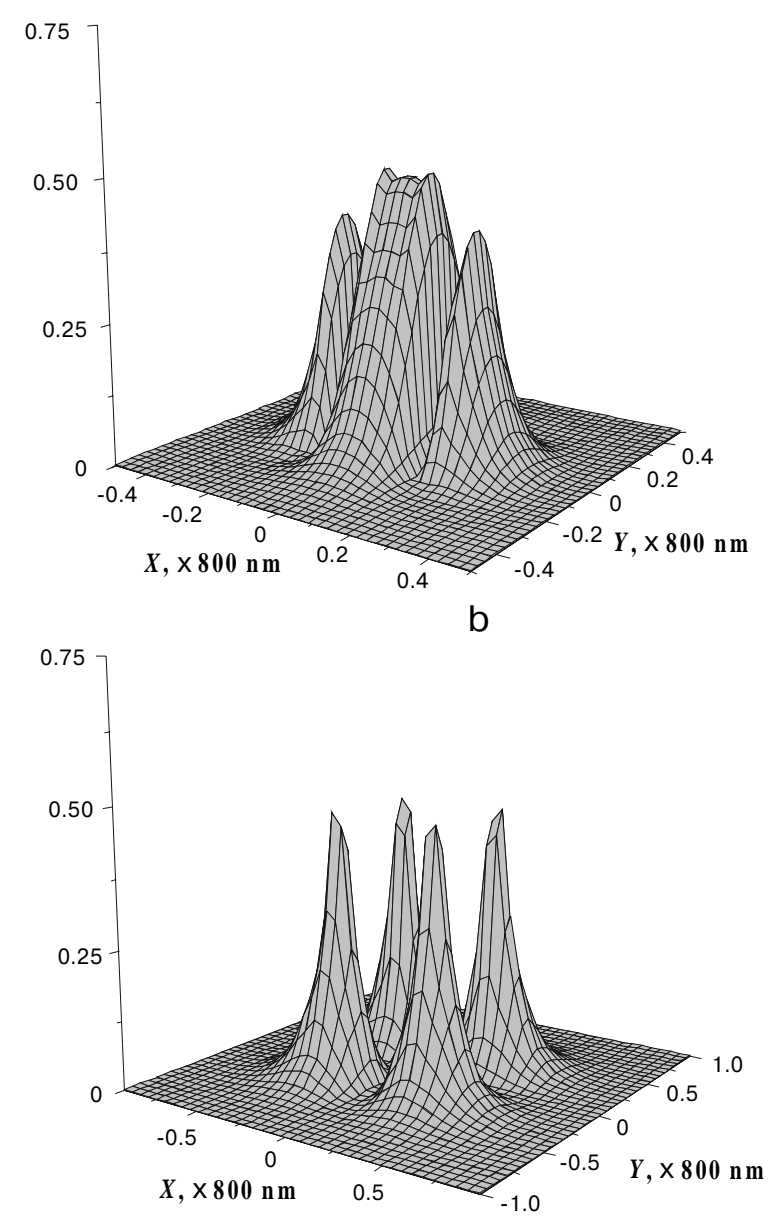

d

Fig.7. The gray scale map (a), (c) representation and $3 \mathrm{~d}$ intencity distribution (b), (d) of near-field image of an rectangular object $\left(200 \times 200 \times 20 \mathrm{~nm}^{3}\right)$ when the distance between a scanned plane and an object is equal to $30 \mathrm{~nm}$. (a), (b) - both external and detected fields are polarized along $\mathrm{O} x$ axes; (c), (d) - the external field is polarized along $\mathrm{O} y$ axes and detected field is polarized along $\mathrm{O} x$ axes.

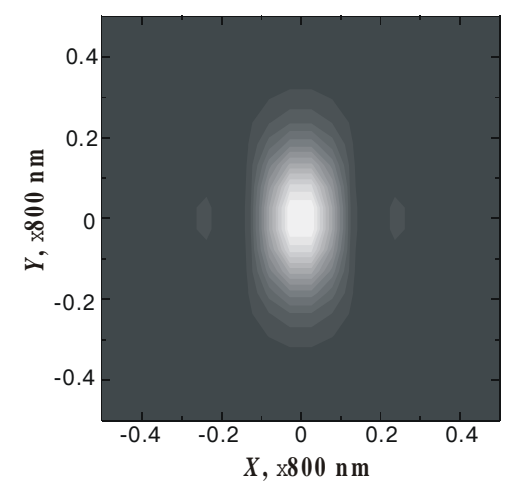

a

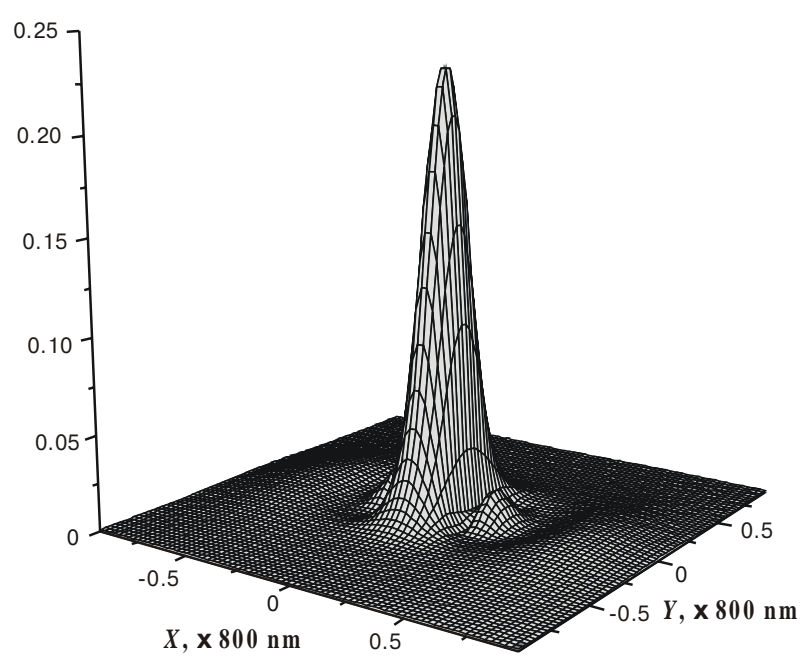

b

Fig.8a,b. The same as in Fig.6, but calculated for a distance between a scanned plane and an object $l=90 \mathrm{~nm}$. 


\section{Lozovski, S. Bozhevolnyi: The exact solution of self-consistent equations...}

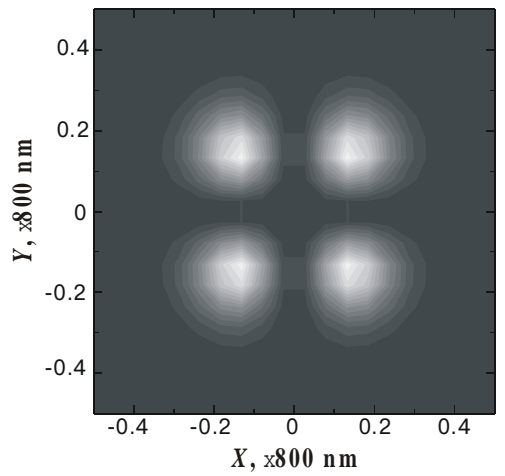

C

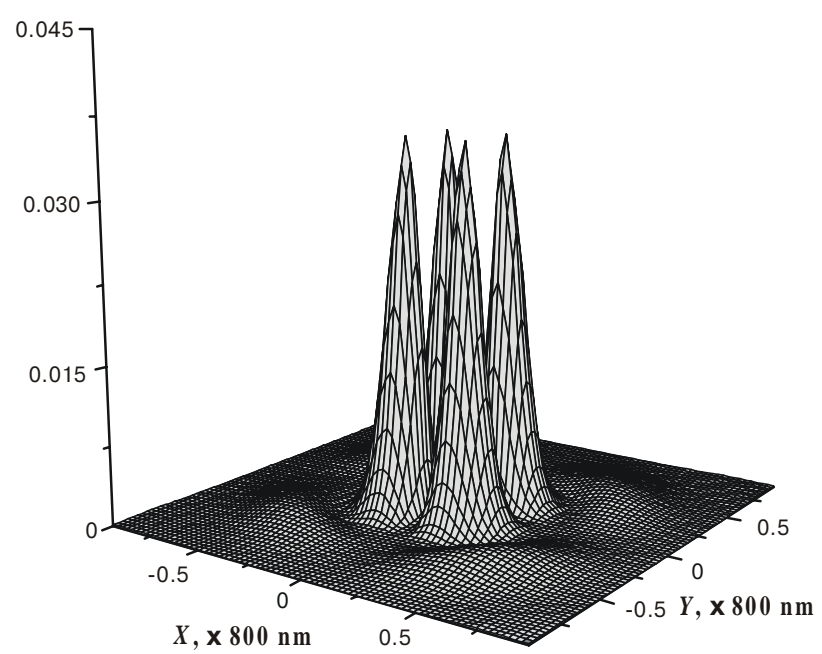

d

Fig.8c,d. The same as in Fig.6, but calculated for a distance between a scanned plane and an object $l=90 \mathrm{~nm}$.

\section{References}

1. D.W.Pohl and D.Courjon, eds., Near Field Optics (Kluwer, Dordrecht, The Netherlands, 1993).

2. S.I.Bozhevolnyi, B.Vohnsen and K.Pedersen, Near-field optical microscopy of nonlinear susceptibilities // Opt.Commun. 150, pp.4955 (1998).

3. S.I.Bozhevolnyi, K.Pedersen, T.Skettrup, X.Zhang and M.Belmonte, Far- and near-field second-harmonic imaging of ferroelectric domain walls // Opt. Commun. 152, pp.221-224 (1998).

4. J.-J.Greffet and R.Carminati, Image formation in near-field optics // Progr. in Surf. Sci., 56, pp.133-237 (1997).

5. Ole Keller, Mufei Xiao and Sergey Bozhevolnyi, Configurational resonances in optical near-field microscopy: a rigorous point-dipole approach // Surf. Sci. 280, pp.217-230 (1993).

6. Ansheng Liu and Garnett W. Bryant, Near-field second-harmonic generation of semiconductor quantum dots // Phys. Rew. B 59, pp. 2245-2253 (1999).

7. A. Lakhtakia, Macroscopic theory of the coupled dipole approximation method // Opt. Commun., 79, pp. 1-5 (1990).

8. L. Novotny, Allowed and forbidden light in near-field optics. I. A single dipolar light source // J. Opt. Soc. Am. A 14, pp. 91-104 (1997) Allowed and forbidden light in near-field optics. II. Interacting dipolar particles // J. Opt. Soc. Am. A 14, pp. 105-113 (1997).

9. M.Xiao, S.Bozhevolnyi, Imaging with reflection near-field optical microscope: contributions of middle and far fields // Opt. Commun., 130, pp. 337-347 (1996).

10. M.Xiao, Theoretical treatment for scattering scanning near-field optical microscopy // J.Opt.Soc.of Am. A 14, pp. 2977-2984 (1997).

11. C.Girard, D.Courjon, The role of scanning mode in near-field optical microscopy // Surf.Sci., 382, pp. 9-18 (1997)

12. O.Keller, Local fields in the electrodynamics of mesoscopic media // Phys. Rep. 268, pp.85-262 (1996).

13. V.Lozovski, Local field equations in electro-dynamics of systems of small particles / / Opt. and Spectroscopy, 86, pp.96-101 (1999).
14. Yasushi Ohfuti and Kikuo Cho, General separability of linear and non-linear optical susceptibilities // Phys. Rev. B, 52, pp.4828-4832 (1995).

15. E.M.Lifshitz and L.P.Pitaevskii, Statistical Physics, Part 2 (Course of Theoretical Physics: 9) (Oxford, Pergamon Press, 1980).

16. A.A.Abrikosov, L.P.Gor'kov and I.Ye.Dzyaloshinskii, Quantum Field Theoretical Methods in Statistical Physics, (Oxford, Pergamon Press, 1965).

17. H.M.Nussenzveig, Causality and analyticity in optics // AIP Conference Proceedings, issue. 65, pp.9-30 (1980).

18. B.I.Khudik, V.Z.Lozovski, A.F.Zhuravlev, Optical theorem in ultrathin film electrodynamics // Phys. Stat. Solidi (b), 151, pp. 111-119 (1989).

19. M.A.Karam, Polarimetric optical theorem // J.Opt.Soc.of Am. A 15, pp. 196-201 (1998)

20. Yu.A.Baloshin, A.V.Kostin, The optical theorem for light scattering from a dielectric rough surface // Opt. Commun. 160, pp.22-26 (1999).

21. O.Keller, Quantum dots of light // J.of Nonlinear Opt. Phys. and Mater., 5, pp.109-132 (1996).

22. Jun Miyake, Masayuki Hara, Protein-based nanotechnology: molecular construction of proteins // Mater. Sci. Ing. C 4 , pp.213-219 (1997).

23. C.Nicolini, Supramolecular architecture and molecular bioelectronics // Thin Solid Films, 284-285, pp.1-5 (1996).

24. U.Heinzmann, S.Holloway, A.W.Kleyn, R.E.Palmer and K.J.Snowdon, Orientation in molecule-surface interactions // J.Phys.: Condens. Matter., 8, pp.3245-3269 (1996).

25. Y.Majima, K.Kato, M.Iwamoto, K.Ichimura, Investigation of dynamic orientation process of nematic liquid crystals triggered by conformation change of surface monolayer // IEICE Transactions on Electronics, E81-C, pp.1070-1076 (1998).

26. J van Bladel, Some remarks on Green's dyadic for infinite space // IRE Trans. Antennas Propagat., AP-9, pp. 563-566 (1961).

27. A.D.Yaghjian, Electric dyadic Green's functions in the source region // Proceed. of the IEEE, 68, pp.248-263 (1980).

28. K.M.Chen, A simple physical picture of ten-sor Green's function in source region // Proceed. of the IEEE, 65, pp.1202-1204 (1977). 